\title{
Which dressing do donor site wounds need?: study protocol for a randomized controlled trial
}

\author{
Anne M Eskes ${ }^{1,2 \dagger}$, Fleur E Brölmann ${ }^{1 \dagger}$, Louise AA Gerbens ${ }^{1}$, Dirk T Ubbink ${ }^{1,3^{*}}$ and Hester Vermeulen ${ }^{1,4}$, for \\ The REMBRANDT study group (REMBRANDT)
}

\begin{abstract}
Background: Donor site wounds after split-skin grafting are rather 'standard' wounds. At present, lots of dressings and topical agents for donor site wounds are commercially available. This causes large variation in the local care of these wounds, while the optimum 'standard' dressing for local wound care is unclear. This protocol describes a trial in which we investigate the effectiveness of various treatment options for these donor site wounds.
\end{abstract}

Methods: A 14-center, six-armed randomized clinical trial is being carried out in the Netherlands. An a-priori power analysis and an anticipated dropout rate of $15 \%$ indicates that 50 patients per group are necessary, totaling 300 patients, to be able to detect a $25 \%$ quicker mean time to complete wound healing. Randomization has been computerized to ensure allocation concealment. Adult patients who need a split-skin grafting operation for any reason, leaving a donor site wound of at least $10 \mathrm{~cm}^{2}$ are included and receive one of the following dressings: hydrocolloid, alginate, film, hydrofiber, silicone dressing, or paraffin gauze. No combinations of products from other intervention groups in this trial are allowed. Optimum application and changes of these dressings are pursued according to the protocol as supplied by the dressing manufacturers. Primary outcomes are days to complete wound healing and pain (using a Visual Analogue Scale). Secondary outcomes are adverse effects, scarring, patient satisfaction, and costs. Outcome assessors unaware of the treatment allocation will assess whether or not an outcome has occurred. Results will be analyzed according to the intention to treat principle. The first patient was randomized October 1, 2009.

Discussion: This study will provide comprehensive data on the effectiveness of different treatment options for donor site wounds. The dressing(s) that will prevail in effectiveness, satisfaction and costs will be promoted among clinicians dealing with such patients. Thus, we aim to contribute a well-designed trial, relevant to all clinicians involved in the care for donor site wounds, which will help enhance uniformity and quality of care for these patients.

Trial registration: http://www.trialregister.nl, NTR1849. Date registered: June 9, 2009

\section{Background}

Split skin grafting (SSG) is a widely used reconstructive technique to repair skin defects (e.g. burns, chronic, and traumatic wounds) [1,2], including those that cannot be covered by a skin flap or are not likely to heal by secondary intention [3]. The wound created after harvesting the skin is called the donor site wound (DSW). Depending on the thickness of the SSG, the DSW should re-epithelialize completely in 7 to 21 days [3]. Optimum local care for

\footnotetext{
* Correspondence: d.ubbink@amc.nl

† Contributed equally

'Quality Assurance \& Process Innovation, Academic Medical Center,

University of Amsterdam, Amsterdam, the Netherlands

Full list of author information is available at the end of the article
}

these DSWs should promote wound healing and be costeffective, while it should prevent complications, such as pain, discomfort, infection, and scarring. Particularly pain and discomfort are reported to occur more frequently from DSWs than at the recipient site [3-5].

Clinical practice shows a large number of dressings and topical agents for DSWs, while the optimum dressing choice for local wound care is unclear $[1,2,6,7]$. Consequently, large variation exists among health care professionals regarding their choice for wound dressing materials or topical agents to treat DSWs [8-10]. Based on national surveys, alginates appear to be the most commonly used primary dressing [8-10], probably due to their additional haemostatic properties $[11,12]$. They are

\section{() Biomed Central}

(c) 2011 Eskes et al; licensee BioMed Central Ltd. This is an Open Access article distributed under the terms of the Creative Commons Attribution License (http://creativecommons.org/licenses/by/2.0), which permits unrestricted use, distribution, and reproduction in any medium, provided the original work is properly cited. 
followed by films, hydrofibers, silicone dressings and paraffin gauzes [8].

Available evidence comprises four systematic reviews (SRs), presenting a lack of strong evidence for the effectiveness of the different dressings for the treatment of DSWs, especially for alginates $[1,2,6,7]$. These SRs tentatively conclude that moist dressings are preferable over non-moist dressings in terms of wound healing. Hydrocolloid and films seem better than nearly all other materials (e.g. alginates, paraffin gauzes, hydrofibers, and foams) as to healing and pain [7]. Hydrofibers in turn seem to outperform tulle dressings in terms of wound healing and pain $[13,14]$. Although tulle dressings seem to be least suitable for the local treatment of DSWs, recent evidence shows that gauze-based dressings still have a place in wound care [15]. Some centers still adhere, or have returned to, these gauzebased dressings [16]. Silicone-based dressings have the advantage of being non-adhesive, although they tend to dislocate easily and do not seem to outperform alginates [17]. These conclusions are formulated cautiously as most authors state that more well designed and rigorous studies are needed.

We therefore conceived a trial to compare the six most promising dressing groups, based on common usage and available evidence. In this paper we will report on the design of our 14-center six-armed randomized clinical trial (RCT). This trial received the acronym "Rembrandt" trial, which stands for Recognizing Effective Materials By Randomizing \& Assessing New Donorsite Treatments. In this trial we aim to answer the following question: Which of the following dressing materials for DSW of SSGs stand out in effectiveness: hydrocolloids, alginates, films, hydrofibers, silicone dressings, or paraffin gauzes, in terms of wound healing, adverse effects (e.g. pain and scarring), and costs?

\section{Methods}

\section{Protocol and registration}

The methods applied in our 14-center RCT were specified in advance, documented in a protocol, and registered (http://www.trialregister.nl, NTR 1849). The study was approved by the local medical ethics committee and by the institutional review boards of each participating hospital or burns centre. The methods used are summarized here according to the revised CONSORT Statement [18].

\section{Design and setting}

We designed a national, 14-center RCT with six treatment groups in the Netherlands (Figure 1). The coordinating center (Academic Medical Center at the University of Amsterdam) invited hospitals (i.e. departments of surgery, plastic surgery and otorhinolaryngology) and burns centers to participate in the trial, resulting in 13 contributing hospitals (4 university hospitals, 5 teaching clinics and 4 general hospitals) and 1 burns center.

\section{Eligibility criteria for patients}

In this trial we include all adult patients, either hospitalized or under treatment in the outpatient clinic in one of the contributing hospitals or burns centre, who need a SSG-operation for any reason. The DSW should have a minimum size of $10 \mathrm{~cm}^{2}$ (to allow proper application and investigation of the study dressings) and be suitable for all treatment options in the trial. Patients are included after full, understandable and neutral explanation by the treating physician or coordinating investigators and after giving written informed consent. Patients are excluded when they receive a treatment known to seriously impair normal wound healing (e.g. chemotherapy, corticosteroids, or local irradiation therapy) or if patients are not physically or mentally able to consent.

\section{Interventions}

Before starting the trial, manufactures of the products involved were invited to develop a protocol how best to apply their wound materials for DSWs, to ensure correct and uniform application of the six different dressing groups:

1. A paraffin gauze-based material (e.g. Jelonet ${ }^{\circledR}$, Adaptic $\left.{ }^{\circledR}\right)$;

2. A hydrocolloid (e.g. DuoDERM ${ }^{\circledR}$ E);

3. An alginate (e.g. Kaltostat ${ }^{\circledR}$, Algisite $^{\circledR}$, Melgisorb $\left.{ }^{\circledR}\right)$;

4. A semi-permeable film (e.g. Tegaderm ${ }^{\circledR}$, Opsite ${ }^{\circledR}$ );

5. A silicone dressing (e.g. Mepitel $\left.{ }^{\circledR}\right)$;

6. A hydrofiber (e.g. Aquacel ${ }^{\circledR}$ ).

The coordinating investigators (FEB and AME) or dressing manufactures orally instructed medical and nursing staff on the wards and out-patient clinics of the contributing centers at the beginning of the trial. Furthermore, they received written application advices as reminders for a uniform treatment protocol, e.g. regarding change frequency and treatment duration. Posters and pocket charts were also distributed to inform about inclusion criteria and contact persons.

\section{Surgical procedure}

A SSG operation is to be performed with an electric or pneumatic dermatome or free hand-knife, according to local best practice. The SSG should preferably be between 0.20 and $0.30 \mathrm{~mm}$ to achieve a reasonably uniform depth of the DSW and should be taken from thighs, arms or buttocks. Method of hemostasis of the DSW is at the discretion of the surgeon (e.g. adrenaline-soaked gauze). However, the decision to use hemostasis must be made before randomization and will be recorded. 


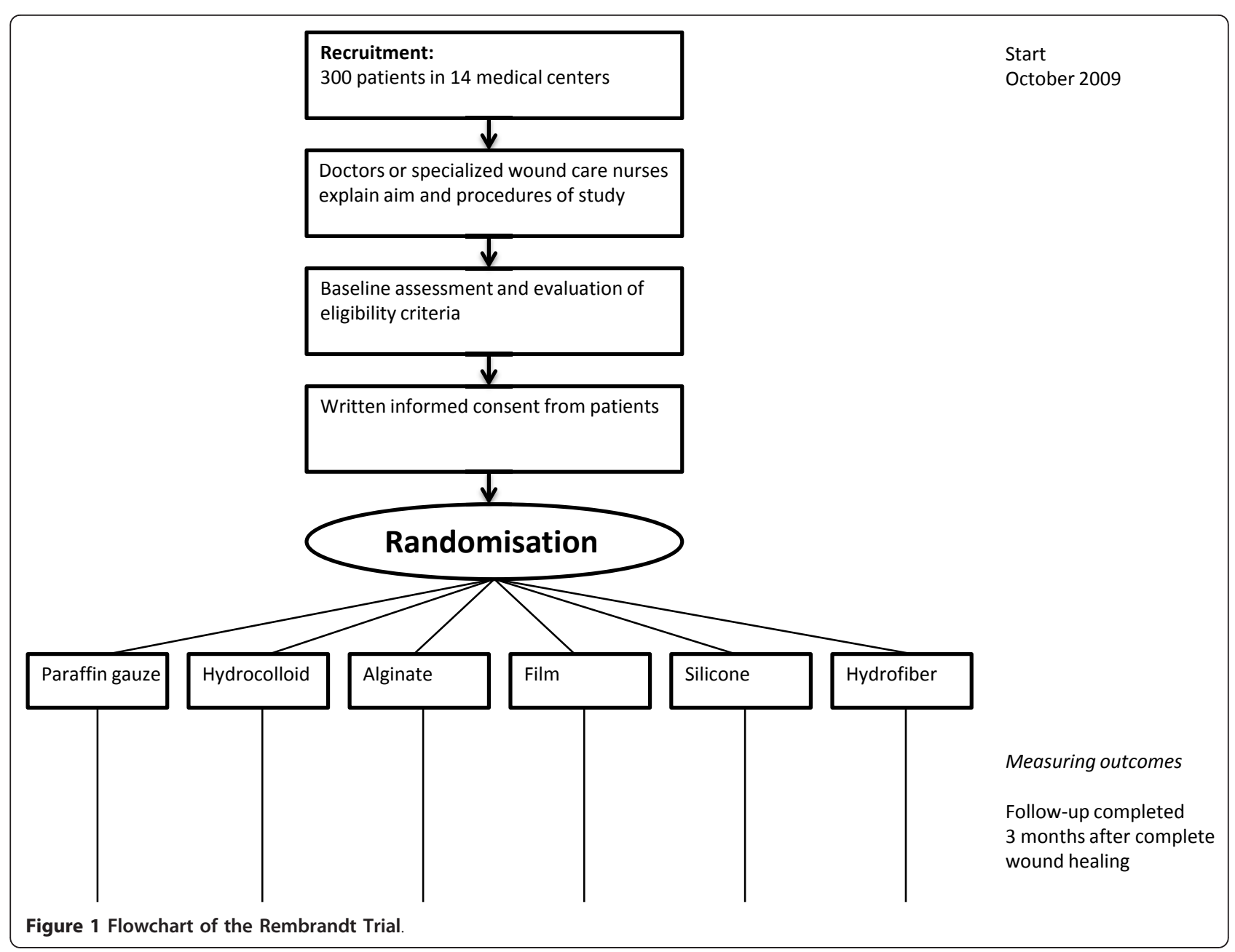

\section{Wound treatment}

Local wound care according to the assigned dressing group starts directly after randomization (see heading Randomization). The brand of the dressing will be recorded. No combinations of products from other dressing groups in this trial are allowed to ensure that the effect found after completion of the trial can be attributed only to the dressing to which the patient was allocated. The optimum changing frequency will be pursued as advised for each dressing material. This may differ from no dressing changes (e.g. hydrofiber) to daily changes in case of leakage (e.g. hydrocolloid or paraffin gauze). We allow coverage of the primary dressings with cotton gauzes and bandages. The type of secondary dressings used will be recorded. Furthermore, we will record any crossovers and make sure the patient returns to the initially allotted dressing.

\section{Co-interventions}

Additional wound debridement, cleansing or protection may be indicated during a dressing change and is allowed in all treatment arms, as this reflects real life. In case of an (impending) wound infection, the wound may be treated with iodine (Povidone- or Cadexomer iodine), to be applied beneath the allotted dressing material [19]. The type of iodine used will be recorded.

\section{Study outcomes \\ Primary endpoints}

The primary endpoint with respect to the effectiveness of wound dressings in the treatment of DSWs is time to complete wound healing. We define wound healing as reepithelialization of the total wound surface. We decided this is not the case until all crusts have come off. This endpoint is to be assessed by an independent investigator who is not aware of the treatment given. The second primary outcome is pain from the donor site area. It is documented by the patient on a Visual Analogue Scale (VAS), varying from 0 (no pain) to 10 (intolerable pain). This is scored daily for the first two weeks postoperatively and twice a week during the third and fourth week, in a patient-held diary. Both primary endpoints are meaningful and relevant to patients and were therefore used for the sample size calculation [20]. 


\section{Secondary endpoints}

As secondary endpoints we assess the occurrence of local complications, e.g. wound infections, based on clinical symptoms of infection, scarring at 12 weeks postoperatively (using Patient and Observer Scar Assessment Scale (POSAS) assessed by the patients themselves and researchers, treating physicians or specialized wound nurses) [21], patient satisfaction (varying from 1 (absolutely dissatisfied) to 10 (absolutely satisfied), and costs (material and nursing costs). Itching scores are also collected by using a VAS, ranging from 0 (no itching) to 10 (intolerable itching) and obtained through the patient-held diary.

\section{Randomization}

Patients are to be randomized in the operation theatre, just after the skin harvest and hemostasis, and before the DSW is to be dressed. In each contributing center an appointed officer performs the randomization using an online computer software program (ALEA NKI-AVL, Amsterdam, The Netherlands, Release: 2.2.) to ensure allocation concealment. The trial is stratified by center, with a balanced allocation ratio for each treatment arm using a biased coin [22]. The biased coin method preserves most of the unpredictability associated with simple randomization [22].

\section{Blinding}

Blinding of patients and care-providers (e.g. doctors and nurses) is not possible because the treatment options cannot be masked. To overcome this possible source of performance bias, independent doctors and nurses of the outpatient clinic, who are unaware of the treatment allocation, will assess whether or not an endpoint has occurred [23].

\section{Sample size}

The study size calculation (using nQuery Advisor version 7.0, Statistical Solutions Ltd, Cork, Ireland) was based on two primary outcomes using a one-way analysis of variance. To detect a $25 \%$ quicker mean wound healing, which is in agreement with the study of Wiechula [2], and with a $5 \%$ significance level, a power of $90 \%$, and a standard deviation (SD) of 3 days, a sample size of 50 patients per group is necessary, given an anticipated dropout rate of $15 \%$. This number also allows detection of a minimum difference in pain scores of 2.0 or greater (with a SD of 2) on the VAS and discernment of a cost difference of $€ 2$ per day. To recruit this number of patients an 18-month inclusion period is anticipated based on the performed number of SSGs as estimated by each of the 14 co-operating hospitals.

\section{Data collection}

The coordination center designed a standardized case record form (CRF) and distributes this in a paper-based or electronic version. The latter one is made available through a secured website, http://www.rembrandt-trial. $\mathrm{nl}$ (using Joomla, an open-source website software package), which also facilitates remote patient randomization and data entry. We collect copies of all completed forms from the co-operating hospitals and maintain the database using SPSS software (PASW statistics version 18.0, IBM, Armonk, NY, USA). Data are collected on baseline demographic and clinical patient characteristics of each group, whether the patients receive the allotted treatment and complete the study protocol, and are analyzed for the primary outcomes. Patients whose treatment deviates from the initial allocation will be described together with the reasons for this. Data on all important adverse events or side effects in each intervention group are recorded as well. Data from the patient-held diaries will be returned to the coordinating center. Double data entry will be conducted by FEB and AME, and compared using the SPSS Data Entry Builder program (Release 4.0.2). We will resolve any discrepancies by discussion and by re-checking the data.

\section{Data monitoring}

Data completeness is reviewed weekly, and reminders or queries are sent timely. FEB and AME visit the cooperating hospitals on a regular basis to promote the trial and to be closely associated with the data collection. Thus, an accurate and complete data set is ensured. Because no (serious) adverse effects were expected from the commercially available dressings that would require interim analysis, we refrained from installing a data safety monitoring board.

\section{Data analysis}

Data coding and analysis will be carried out using SPSS software (PASW statistics version 18.0, IBM, Armonk, NY, USA). Differences in outcome variables will be analyzed on an intention-to-treat basis. A general linear model will be used to analyze the differences between the treatment arms for the various endpoints measured repeatedly, as the data are likely to be unequally distributed. Differences in wound healing time between the dressing groups will be examined using the Kaplan-Meier method and the Mantel-Cox log-rank test. Data analysis will be conducted by the authors and replicated by the Clinical Research Unit of the coordinating hospital. We will record any crossovers and make sure the patient returns to the initially allotted dressing. Missing data are dealt with by using the Generalized Estimating Equations model in our statistical analysis.

\section{Data storage}

Data are stored at the coordinating center in a Trial Master File and at the co-operating hospital sites, where 
an Investigator File is kept. After finishing the trial, data will be saved for at least 5 years, in accordance with the recommendations as to low risk studies of the Dutch Federation of University Medical Centers.

\section{Discussion}

In current clinical practice, a 'standard' wound such as a DSW does not appear to be associated with a uniform dressing choice [8]. Also the systematic reviews of available literature report a large clinical heterogeneity among the available trials $[1,2,6,7]$. To date, available evidence allows the tentative conclusion that dressings creating a moist environment seem to be preferable over gauzebased dressings in the management of DSWs. This recommendation is tentatively formulated because strong recommendations for clinical practice are hard to draw, mainly due to the poor quality and small sample sizes of the available trials. Therefore, most systematic reviews recommend new and large randomized clinical trials $[2,6,7]$.

However, doing research in the realm of wound care involves much more than simply comparing wound care products in eligible patients. It poses many methodological and practical challenges in the design and execution of trials [24].

The first challenge is the design of our, intentionally pragmatic, RCT. To enhance the applicability and generalizability of the results of this trial, we chose a multicenter trial design and recruited patients from low- and high-volume centers like teaching hospitals and burns centers. We realize that for many surgical procedures, patients have better outcomes in high-volume centers [25-29]. However, split-skin grafting is a rather common procedure, also in smaller hospitals. Second, we are forbearing regarding local clinical care, for example by allowing several brands within each dressing group, different depths of skin grafting, and different methods of hemostasis. This helps mimicking 'real life', at the cost of losing some contrast between the six treatment arms.

Although we are liberal and pragmatic at some points, we feel we need to and can be strict in others. We urge the contributing centers to adhere to the same dressing type until complete wound healing is reached. This allows us to appreciate the true effects of each of the dressing types studied. Some argue this is not reflecting common practice [30], in which the dressing type is changed in response to any change in the clinical condition of the wound during the healing process. We do not think this will be a frequently occurring issue since these superficial DSWs usually have fairly short healing times. The protocol does allow for an antiseptic agent to be added in case of an (impending) wound infection.

Another frequent methodological challenge in wound care research is the use of subjective or surrogate outcome variables. In this trial we aim to measure our endpoints in a reliable and valid way. We strictly predefined our primary endpoint, time to complete wound healing. In a previous study it was shown that, by using this strict definition, specialized nurses had a better inter-observer agreement than doctors or nurses regarding the assessment of complete wound healing [31]. Therefore, in this trial predominantly specialized nurses will assess our primary endpoint. Our second primary outcome variable, pain, is measured using VAS scores. This is a reliable and acknowledged scale for general clinical use [32,33].

Today, financial support is a necessity to properly conduct a (multicenter) trial. For this purpose, we obtained funding from an independent institution, i.e. the Dutch Burns Foundation, which is to be preferred over subvention from one or more dressing manufacturers to avoid any publication bias. To avoid any conflict of interest, analysis and reporting of the trial stays the domain of the investigators.

The strengths of this trial are firstly the fruitful collaboration with manufacturers, who developed a dedicated protocol for the treatment of the DSW with their product. This greatly supports the uniform application of each dressing type under study. Second, using this sixarmed, multicenter trial we investigate the effectiveness of the dressings most commonly used in the Netherlands and most promising from the available literature. This will facilitate implementation of the results. We expect to present the results of this trial in the course of 2012.

\section{Financial support}

Unrestricted grant from the Dutch Burns Foundation. They have no say in the analysis and publication of the results of this trial.

\section{The Rembrandt study group}

Academic Medical Center: J.C. Goslings; Martini Hospital Groningen: S.J.M. Jongen; Leiden University Medical Center: J.F.A. van der Werff; operating at that time at University Medical Center Utrecht: A.H. Schuurman; Free University Hospital Amsterdam: F.B. Niessen; OLVG Amsterdam: A.C. Vahl; Kennemer Gasthuis: A.K.J. Ahmed; Spaarne Hospital: D. Nio; Tergooi Hospitals: N. Koedam; Waterland Hospital: P. Heres; Isala Klinieken Zwolle: E.G.J.M. Pierik; Rijnland Hospital; J.F.A. van der Werff; operating at that time at Gelre Hospitals Zutphen; M.L.M.J. Goessens; Lange Land Hospital: L. Levert-Brand.

\section{Abbreviations}

CRF: case-record form; CRU: Clinical Research Unit; CONSORT: Consolidated Standards of Reporting Trials; DSW: donor site wound(s); POSAS: Patient and Observer Scar Assessment Scale; RCT: randomized clinical trial; SD: standard 
deviation; SR: systematic review; SSG: split skin graft(s); VAS: Visual Analogue Scale.

\section{Acknowledgements}

We especially thank the Dutch Burns Foundation for their subvention, which makes this study possible. We also thank the manufactures of all dressing materials involved in this trial for developing a protocol how best to handle their wound materials for the DSW to ensure correct and uniform application. We also like to thank all doctors, specialized wound care nurses, and the ward and outpatient nurses involved in the trial. Their relentless help makes this study possible.

\section{Author details}

'Quality Assurance \& Process Innovation, Academic Medical Center, University of Amsterdam, Amsterdam, the Netherlands. ${ }^{2}$ Amsterdam School of Health Professions, Amsterdam, the Netherlands. ${ }^{3}$ Surgery, Academic Medical Center, University of Amsterdam, Amsterdam, the Netherlands. ${ }^{4}$ Amsterdam School of Health Professions, Amsterdam, the Netherlands.

\section{Authors' contributions}

AME participated in the trial design and drafted the manuscript. FEB participated in the trial design and was involved in drafting the manuscript. LAAG has made substantial contributions to the design of the trial and gave her intellectual input on the manuscript. DTU and HV were involved in designing of the trial, obtained funding for the trial, and gave intellectual input on the manuscript. They are the project leaders and have overall responsibility for the trial. All authors have read and approved the final version of the manuscript.

\section{Competing interests}

The authors declare that they have no competing interests.

Received: 8 August 2011 Accepted: 17 October 2011

Published: 17 October 2011

\section{References}

1. Rakel BA, Bermel MA, Abbott LI, Baumler SK, Burger MR, Dawson CJ, Heinle JA, Ocheltree IM: Split-thickness skin graft donor site care: a quantitative synthesis of the research. Appl Nurs Res 1998, 11(4):174-182.

2. Wiechula R: The use of moist wound-healing dressings in the management of split-thickness skin graft donor sites: a systematic review. Int J Nurs Pract 2003, 9(2):S9-17.

3. Ratner D: Skin grafting. Semin Cutan Med Surg 2003, 22(4):295-305.

4. Persson K, Salemark L: How to dress donor sites of split thickness skin grafts: a prospective, randomised study of four dressings. Scand J Plast Reconstr Surg Hand Surg 2000, 34(1):55-59.

5. Feldman DL: Which dressing for split-thickness skin graft donor sites? Ann Plast Surg 1991, 27(3):288-291.

6. Voineskos SH, Ayeni OA, McKnight L, Thoma A: Systematic review of skin graft donor-site dressings. Plast Reconstr Surg 2009, 124(1):298-306.

7. Schreuder SM, Qureshi MA, Vermeulen H, Ubbink DT: Dressings and topical agents for treating donor sites of split-skin grafts: a systematic review [abstract]. EWMA Journal 2009, 9(3):22.

8. Eskes AM, Gerbens LAA, Ubbink DT, Vermeulen H: [Current treatment policies of donor site wounds after split-skin grafting: a national survey]. Ned Tijdschr Heelkd 2011, 20(2):66-69, [Dutch].

9. Geary PM, Tiernan E: Management of split skin graft donor sites-results of a national survey. J Plast Reconstr Aesthet Surg 2009, 62(12):1677-1683.

10. Lyall PW, Sinclair SW: Australasian survey of split skin graft donor site dressings. Aust N Z J Surg 2000, 70(2):114-116.

11. Blaine G: Alginates in surgery. Med Press 1947, 218(8):166-169.

12. Groves AR, Lawrence JC: Alginate dressing as a donor site haemostat. Ann R Coll Surg Engl 1986, 68(1):27-28.

13. Barnea Y, Amir A, Leshem D, Zaretski A, Weiss J, Shafir R, Gur E: Clinical comparative study of aquacel and paraffin gauze dressing for split-skin donor site treatment. Ann Plast Surg 2004, 53(2):132-136.

14. Lohsiriwat $\mathrm{V}$, Chuangsuwanich $\mathrm{A}$ : Comparison of the ionic silvercontaining hydrofiber and paraffin gauze dressing on split-thickness skin graft donor sites. Ann Plast Surg 2009, 62(4):421-422.

15. Ubbink DT, Vermeulen H, Goossens A, Kelner RB, Schreuder SM, Lubbers MJ: Occlusive vs gauze dressings for local wound care in surgical patients: a randomized clinical trial. Arch Surg 2008, 143(10):950-955

16. Malpass KG, Snelling CF, Tron V: Comparison of donor-site healing under Xeroform and Jelonet dressings: unexpected findings. Plast Reconstr Surg 2003, 112(2):430-439.

17. O'Donoghue JM, O'Sullivan ST, O'Shaughnessy M, O'Connor TP: Effects of a silicone-coated polyamide net dressing and calcium alginate on the healing of split skin graft donor sites: a prospective randomised trial. Acta Chir Plast 2000, 42(1):3-6.

18. Moher D, Hopewell S, Schulz KF, Montori V, Gotzsche PC, Devereaux PJ, Elbourne D, Egger M, Altman DG: CONSORT 2010 explanation and elaboration: updated guidelines for reporting parallel group randomised trials. BMJ 2010, 340:c869.

19. Vermeulen $H$, Westerbos SJ, Ubbink DT: Benefit and harm of iodine in wound care: a systematic review. J Hosp Infect 2010, 76(3):191-199.

20. Vermeulen H, Ubbink DT, de Zwart F, Goossens A, de Vos R: Preferences of patients, doctors, and nurses regarding wound dressing characteristics: a conjoint analysis. Wound Repair Regen 2007, 15(3):302-307.

21. van de Kar AL, Corion LU, Smeulders MJ, Draaijers LJ, van der Horst CM, van Zuijlen PP: Reliable and feasible evaluation of linear scars by the Patient and Observer Scar Assessment Scale. Plast Reconstr Surg 2005, 116(2):514-522.

22. Schulz KF, Grimes DA: Generation of allocation sequences in randomised trials: chance, not choice. Lancet 2002, 359(9205):515-519.

23. Farrokhyar F, Karanicolas PJ, Thoma A, Simunovic M, Bhandari M, Devereaux PJ, Anvari M, Adili A, Guyatt G: Randomized controlled trials of surgical interventions. Ann Surg 2010, 251(3):409-416.

24. Ubbink $\mathrm{D}$, Vermeulen $\mathrm{H}$ : The challenge of using randomized trials in wound healing (Br J Surg 2010; 97: 303-304). Br J Surg 2010, 97(7):1147-1148

25. Birkmeyer JD, Sun Y, Wong SL, Stukel TA: Hospital volume and late survival after cancer surgery. Ann Surg 2007, 245(5):777-783.

26. Gouma DJ, van Geenen RC, van Gulik TM, de Haan RJ, de Wit LT, Busch OR, Obertop H: Rates of complications and death after pancreaticoduodenectomy: risk factors and the impact of hospital volume. Ann Surg 2000, 232(6):786-795.

27. Holt PJ, Poloniecki JD, Gerrard D, Loftus IM, Thompson MM: Meta-analysis and systematic review of the relationship between volume and outcome in abdominal aortic aneurysm surgery. Br J Surg 2007, 94(4):395-403.

28. Hannan EL, Radzyner M, Rubin D, Dougherty J, Brennan MF: The influence of hospital and surgeon volume on in-hospital mortality for colectomy, gastrectomy, and lung lobectomy in patients with cancer. Surgery 2002, 131(1):6-15.

29. Shervin N, Rubash HE, Katz JN: Orthopaedic procedure volume and patient outcomes: a systematic literature review. Clin Orthop Relat Res 2007, 457:35-41.

30. Gottrup F, Apelqvist J: The challenge of using randomized trials in wound healing. Br J Surg 2010, 97(3):303-304.

31. Eskes AM, Gerbens LA, van der Horst CM, Vermeulen H, Ubbink DT: Is the red-yellow-black scheme suitable to classify donor site wounds? An inter-observer analysis. Burns 2011, 37(5):823-827.

32. Carlsson AM: Assessment of chronic pain. I. Aspects of the reliability and validity of the visual analogue scale. Pain 1983, 16(1):87-101.

33. Williamson A, Hoggart B: Pain: a review of three commonly used pain rating scales. J Clin Nurs 2005, 14(7):798-804.

doi:10.1186/1745-6215-12-229

Cite this article as: Eskes et al:: Which dressing do donor site wounds need?: study protocol for a randomized controlled trial. Trials 2011 $12: 229$. 